\title{
Synergistic Effect of Bupleuri Radix and Scutellariae Radix on Adipogenesis and AMP-Activated Protein Kinase: A Network Pharmacological Approach
}

\author{
Jueun Lee, ${ }^{1}$ Jinbong Park $\left(\mathbb{D},{ }^{1,2}\right.$ Hyewon Park, ${ }^{3}$ Dong-Hyun Youn, ${ }^{1,2}$ Jaehoon Lee, ${ }^{4}$ \\ Seokbeom Hong, ${ }^{4}$ and Jae-Young Um $\mathbb{D}^{1,2}$ \\ ${ }^{1}$ Department of Pharmacology, College of Korean Medicine, Kyung Hee University, Seoul, Republic of Korea \\ ${ }^{2}$ Basic Research Laboratory for Comorbidity Regulation, Comorbidity Research Institute, Kyung Hee University, \\ Seoul, Republic of Korea \\ ${ }^{3}$ Department of Biological Sciences in Korean Medicine, Graduate School, Kyung Hee University, Seoul, Republic of Korea \\ ${ }^{4}$ Department of Korean Medicine, Graduate School, Kyung Hee University, Seoul, Republic of Korea \\ Correspondence should be addressed to Jae-Young Um; jyum@khu.ac.kr
}

Received 20 April 2018; Revised 11 July 2018; Accepted 26 July 2018; Published 23 August 2018

Academic Editor: Hilal Zaid

Copyright (C) 2018 Jueun Lee et al. This is an open access article distributed under the Creative Commons Attribution License, which permits unrestricted use, distribution, and reproduction in any medium, provided the original work is properly cited.

\begin{abstract}
Obesity has become a major health threat in developed countries. However, current medications for obesity are limited because of their adverse effects. Interest in natural products for the treatment of obesity is thus rapidly growing. Korean medicine is characterized by the wide use of herbal formulas. However, the combination rule of herbal formulas in Korean medicine lacks experimental evidence. According to Shennong's Classic of Materia Medica, the earliest book of herbal medicine, Bupleuri Radix (BR) and Scutellariae Radix (SR) possess the Sangsoo relationship, which means they have synergistic features when used together. Therefore these two are frequently used together in prescriptions such as Sosiho-Tang. In this study, we used the network pharmacological method to predict the interaction between these two herbs and then investigated the effects of BR, SR, and their combination on obesity in 3T3-L1 adipocytes. BR, SR, and BR-SR mixture significantly decreased lipid accumulation and the expressions of two major adipogenic factors, peroxisome proliferator-activated receptor-gamma (PPAR $\gamma$ ) and CCAAT/enhancerbinding protein-alpha $(\mathrm{C} / \mathrm{EBP} \alpha)$, and their downstream genes, Adipoq, aP2, and Lipin1 in 3T3-L1 cells. In addition, the BR-SR mixture had synergistic effects compared with BR or SR on inhibition of adipogenic-gene expressions. BR and SR also inhibited the protein expressions of PPAR $\gamma$ and $\mathrm{C} / \mathrm{EBP} \alpha$. Furthermore, the two extracts successfully activated AMP-activated protein kinase alpha (AMPK $\alpha$ ), the key regulator of energy metabolism. When compared to those of BR or SR, the BR-SR mixture showed higher inhibition rates of PPAR $\gamma$ and $\mathrm{C} / \mathrm{EBP} \alpha$, along with higher activation rate of AMPK. These results indicate a new potential antiobese pharmacotherapy and also provide scientific evidence supporting the usage of herbal combinations instead of mixtures in Korean medicine.
\end{abstract}

\section{Introduction}

The epidemiology of obesity is constantly increasing, becoming a health threat all over the world. The World Health Organization has reported that over 1.4 billion adults worldwide are overweight $\left(\mathrm{BMI} \geq 25 \mathrm{~kg} / \mathrm{m}^{2}\right.$ ) or obese (BMI $\geq 30 \mathrm{~kg} / \mathrm{m}^{2}$ ) [1]. However, there are only 5 treatments (orlistat, lorcaserin, liraglutide, phentermine/topiramate, and bupropion/naltrexone) approved by the United States Food and Drug Administration. Despite their efficacies, adverse effects of these drugs lead to search for other options [2].

Obesity is caused by a simple process: when energy intake exceeds expenditure, the excess energy is saved in as a form of lipid. On the other hand, in the perspective of selecting treatments, there are more than pathways to resolve this metabolic syndrome. Pharmacological approach for obesity treatment can be subcategorized into two methods: inhibiting lipid accumulation or consuming accumulated lipids. Former 
method inhibits lipid accumulation in adipose tissues by decreasing appetite, lowering fat absorption, and inhibiting adipogenesis, and the latter is by increasing energy expenditure via fatty acid oxidation or brown adipose tissue- (BAT-) related thermogenesis [3]

Network pharmacology is a system biology-based methodology for pharmacological research $[4,5]$. Network pharmacological research replaces the dominant paradigm of drug design based on "one gene, one drug, one disease," into multitarget drugs that act on biological networks. These unique characteristics of network pharmacology expand the potential use of traditional herbal medicine including Korean medicine, Japanese Kampo medicine, and Traditional Chinese Medicine [6]. In this premise, as obesity is a multipathway-involved disease, and the multicomponent-multitarget herbal drugs may provide an answer to its treatment.

Bupleuri Radix (BR) and Scutellariae Radix (SR) are both components of Sosiho-Tang, a traditional Korean herbal formula which is originally used to treat chronic herbal diseases. The antiobese effect of Sosiho-Tang is reported in a study using 3T3-L1 adipocytes and high fat diet- (HFD-) induced obese C57BL/6J mice [7]. In the current study, Yoo et al. show Sosiho-Tang can inhibit adipogenesis by suppressing CCAAT/enhancer-binding protein-alpha $(\mathrm{C} / \mathrm{EBP} \alpha)$ and peroxisome proliferator-activated receptor-gamma (PPAR $\gamma)$, two key regulators of adipogenesis in vivo and in vitro. Furthermore, other studies demonstrate that BR can attenuate obesity in rats by regulating adipogenic factors [8], while its active compound saikosaponin A inhibits inflammatory markers in adipocytes [9]. Several reports also demonstrate the antiobese and antiadipogenic effect of SR [10] and its active compounds such as baicalin [11-14], baicalein $[15,16]$, and wogonin [17-19].

Based on these studies, we expected that BR and SR are the major components of Sosiho-Tang responsible for antiobese effects. They are used for specific types of obesity patients after diagnosis by a Korean medicine practitioner, and, in addition, several experimental studies also report the possibility. According to Shennong's Classic of Materia Medica, a classic of oriental medicine introducing 365 kinds of herbs and their features [20], BR and SR are Sangsoo (synergistic) pairs. As a pair of Sangsoo (synergism), our hypothesis herein is that BR and SR may show synergistic effects in obesity-related mechanisms. Therefore, in this study, we evaluate the synergistic potential between BR and SR by the network pharmacological approach and assess their effects on obesity by investigating related mechanisms.

\section{Materials and Methods}

2.1. Reagents. 3-Isobutylmethylxanthine (IBMX), dexamethasone (Dex), and Insulin were purchased from Sigma Chemicals (St Louis, MO, USA). Dulbecco's modified Eagle's medium (DMEM), penicillin-streptomycin, bovine serum (BS), and fetal bovine serum (FBS) were purchased from Gibco BRL (Grand Island, NY, USA).
TABLE 1: Nineteen active main components of Scutellariae Radix and Bupleuri Radix.

\begin{tabular}{lc}
\hline Scutellariae Radix & Bupleuri Radix \\
\hline (S)-camphor & $(-)$-alpha-Pinene \\
apigenin & $(\mathrm{Z}, \mathrm{Z})$-farnesol \\
baicalein & Baicalin \\
Baicalin & beta-Eudesmol \\
beta-sitosterol & coumarin \\
scutellarein & kaempferol \\
palmitic acid & quercetin \\
wogonin & capsaicin \\
Linolenic acid methyl ester & saikosaponin a \\
& oleanolic acid \\
\hline
\end{tabular}

2.2. Preparation of Water Extracts of $B R$ and SR. BR (the root of Bupleurum falcatum Linne) and SR (the root of Scutellaria baicalensis Georgi), both originated from China, were purchased from Omniherb Co. (Daegu, Republic of Korea) identified by a specialist of herbs. $100 \mathrm{~g}$ of BR was extracted in $1000 \mathrm{ml}$ of distilled water at $100^{\circ} \mathrm{C}$ for $4 \mathrm{~h} .100$ $\mathrm{g}$ of SR was extracted by the same method. Then, the water extracted BR and SR were freeze dried. The yields were $15.9 \%$ and $17.6 \%$ for BR and SR, respectively. The same amounts of $\mathrm{BR}$ and SR were mixed to prepare the BR-SR mixture.

2.3. Network Pharmacological Analysis. The herbal compound-target gene information was analyzed using the Traditional Chinese Medicine Systems Pharmacology Database (TCMSP). The major chemical components of SR and BR were collected and extracted from TCMSP. We collected each 99 and 147 compounds that encompassed the main components of SR and BR, taking into account the relevant reports. Then we selected the 9 and 10 chemical compounds that satisfied an oral bioavailability $(\mathrm{OB}) \geq 10 \%$ and druglikeness $(\mathrm{DL}) \geq 0.04$ and provide further extraction and optimization of the medicinal ingredients. Nineteen pharmacological ingredients that satisfied the above conditions are showed in Table 1. In a biological network, nodes are visual representations proteins, genes, or metabolites. An edge is a visual representation of a relation. It is a line that connects two nodes and represents biological relationships, such as physical interactions or gene expression regulation [21]. In this study, nodes mean compounds and targets, and edges are relationships between compounds and target. Degree is the number of connected nodes.

To construct a compound-target network, we used Cytoscape 3.6.0 software. By compound-target network, we studied Sangsoo relationships between SR and BR. Multiple targets that participate in 3T3-L1 adipocytes were shared by each compound of SR and BR.

2.4. Cell Culture and Adipocyte Differentiation. 3T3-L1 preadipocytes (American Type Culture Collection, Rockville, MD, USA) was cultured and differentiated as previously described [22]. Briefly, 3T3-L1 preadipocytes were cultured in DMEM containing $1 \%$ penicillin-streptomycin and $10 \%$ 
BS in 6-well plates until confluence at $37^{\circ} \mathrm{C}$. Two days after $100 \%$ confluence (day 0 ), cells were differentiated for $48 \mathrm{~h}$ in differentiation medium composed of DMEM containing $10 \%$ FBS and differentiation inducers (MDI: 0.5 mM IBMX, $1 \mu \mathrm{M}$ Dex, and $1 \mu \mathrm{g} / \mathrm{ml}$ insulin). From day 2 to day 4 , the cells were incubated in culture medium (DMEM plus 10\% FBS and 1 $\mu \mathrm{g} / \mathrm{ml}$ insulin) supplemented with BR, SR, or BR-SR mixture. Then, at day 4 , the medium was changed into fresh culture medium and the cells were cultured for $48 \mathrm{~h}$.

2.5. Cell Viability Assay. Cytotoxicity of BR, SR, and BR-SR mixture was evaluated using the MTS assay, as previously described [23]. The absorbance was measured at $490 \mathrm{~nm}$ using a VERSAmax microplate reader (Molecular Devices, Sunnyvale, CA, USA).

2.6. Oil Red O Staining. Intracellular lipid accumulation was measured by Oil Red $\mathrm{O}$ staining as previously described [24]. The absorbance, which is proportional to intracellular lipid, was measured at $500 \mathrm{~nm}$ with a VERSAmax microplate reader (Molecular Devices, Sunnyvale, CA, USA).

2.7. RNA Isolation and Real-Time Reverse-Transcription Polymerase Chain Reaction (RT-PCR). Total RNA was extracted from BR, SR, or BR-SR mixture-treated cells using QIAzol lysis reagent (Qiagen Sciences Inc., Germantown, MD, USA) and GeneAll RiboEx Total RNA extraction kit (GeneAll Biotechnology, Seoul, Republic of Korea) as previously described [25]. mRNA evaluation was performed using a StepOnePlus Real-time RT-PCR System (Applied Biosystems, Foster City, CA, USA). Primer sequences used in this study are described in Table 2.

2.8. Protein Extraction and Western Blot Analysis. BR, SR, or BR-SR mixture-treated cells were harvested and lysed in Cell Lysis Buffer (Cell Signaling Technology Inc., Danvers, MA, USA). Determination of total protein concentration and western blot analysis were performed as described previously $[25,26]$.

2.9. Statistical Analysis. Results are expressed as mean \pm standard error mean (SEM). Student $t$-test was used to determine statistical relevance. All statistical analyses were performed using GraphPad Prism 5 (GraphPad Software, La Jolla, CA, USA).

\section{Results}

3.1. Compound-Compound Target Network Analysis of BR and $S R$. For prediction analysis of Sangsoo relationship between $\mathrm{BR}$ and SR, we constructed the compound-target network (Figure 1(a)) based on data mining. This network consists of 19 compounds and 504 candidate targets in which pink circles and blue ones correspond to active components and targets, respectively. In the relationships between compounds and targets, we found 265 nodes and 504 edges. The means of degree value (the number of associated targets) of the compounds were 18 , indicating that most of the compounds
TABle 2: Primer sequences $\left(5^{\prime}\right.$ to $\left.3^{\prime}\right)$ for real-time RT-PCR.

\begin{tabular}{lc}
\hline Genes & $5^{\prime}$ to $3^{\prime}$ Oligonucleotide Sequences \\
\hline Mouse Pparg & \\
Sense (Forward) & TTT TCA AGG GTG CCA GTT TC \\
Antisense (Reverse) & TTA TTC ATC AGG GAG GCC AG \\
\hline Mouse Cebpa & \\
Sense (Forward) & GCC GAG ATA AAG CCA AAC AA \\
Antisense (Reverse) & CCT TGA CCA AGG AGC TCT CA \\
\hline Mouse aP2 & \\
Sense (Forward) & CGTAAATGGGGATTTGGTCA \\
Antisense (Reverse) & TCGACTTTCCATCCCACTTC \\
\hline Mouse Adipoq & \\
Sense (Forward) & AGACCTGGCCACTTTCTCCTCATT \\
Antisense (Reverse) & AGAGGAACAGGAGAGCTTGCAACA \\
\hline Mouse Lipin1 & \\
Sense (Forward) & TTCCTTGTCCCTGAACTGCT \\
Antisense (Reverse) & TGAAGACTCGCTGTGAATGG \\
\hline Mouse Gapdh & \\
Sense (Forward) & AAC TTT GGC ATT GTG GAA GG \\
Antisense (Reverse) & GGA TGC AGG GAT GAT GTT CT \\
\hline
\end{tabular}

regulated multiple targets to exert various therapeutic effects. The mean compounds of each target were 6 , showing that most of the targets can interact with multiple compounds simultaneously.

Among the active compounds, quercetin of BR exhibits the highest number of target candidate target interactions (degrees $=154)$, followed by apigenin from SR (degrees $=80$ ), kaempferol from BR (degrees = 59), and wogonin from SR (degrees $=52)$.

In the 504 targets of active compounds, prostaglandin $\mathrm{G} / \mathrm{H}$ syntheses 2 showed the highest degree (degrees = 12), which was followed by apoptosis regulator $\mathrm{Bcl}-2$ and caspase-3 (degrees $=10$ ), prostaglandin $\mathrm{G} / \mathrm{H}$ synthase 1 and phosphatidylinositol-4,5-bisphosphate 3-kinase catalytic subunit, and gamma isoform (degrees $=9$ ). The network pharmacological analysis result demonstrates the potential synergistic effect of SR and BR on adipogenesis and energy expenditure through modulating these relevant proteins.

3.2. $B R$ and SR Inhibit Lipid Accumulation in 3T3-L1 Adipocytes. First, we evaluated the cytotoxicity of BR and SR on 3T3-L1 adipocytes. As shown in Figure 2(a), BR and SR did not affect cell viability up to concentration of $10 \mu \mathrm{g} / \mathrm{ml}$ and $1 \mu \mathrm{g} / \mathrm{ml}$, respectively, when analyzed by an MTS assay. Further experiments were conducted using concentrations which did not decrease cell viability. Next, to evaluate the effect of BR and SR on lipid accumulation, Oil Red O staining assay was performed. Adipocytes treated with BR and SR both showed concentration-dependent decrease of intracellular lipids (Figure 2(b)). 


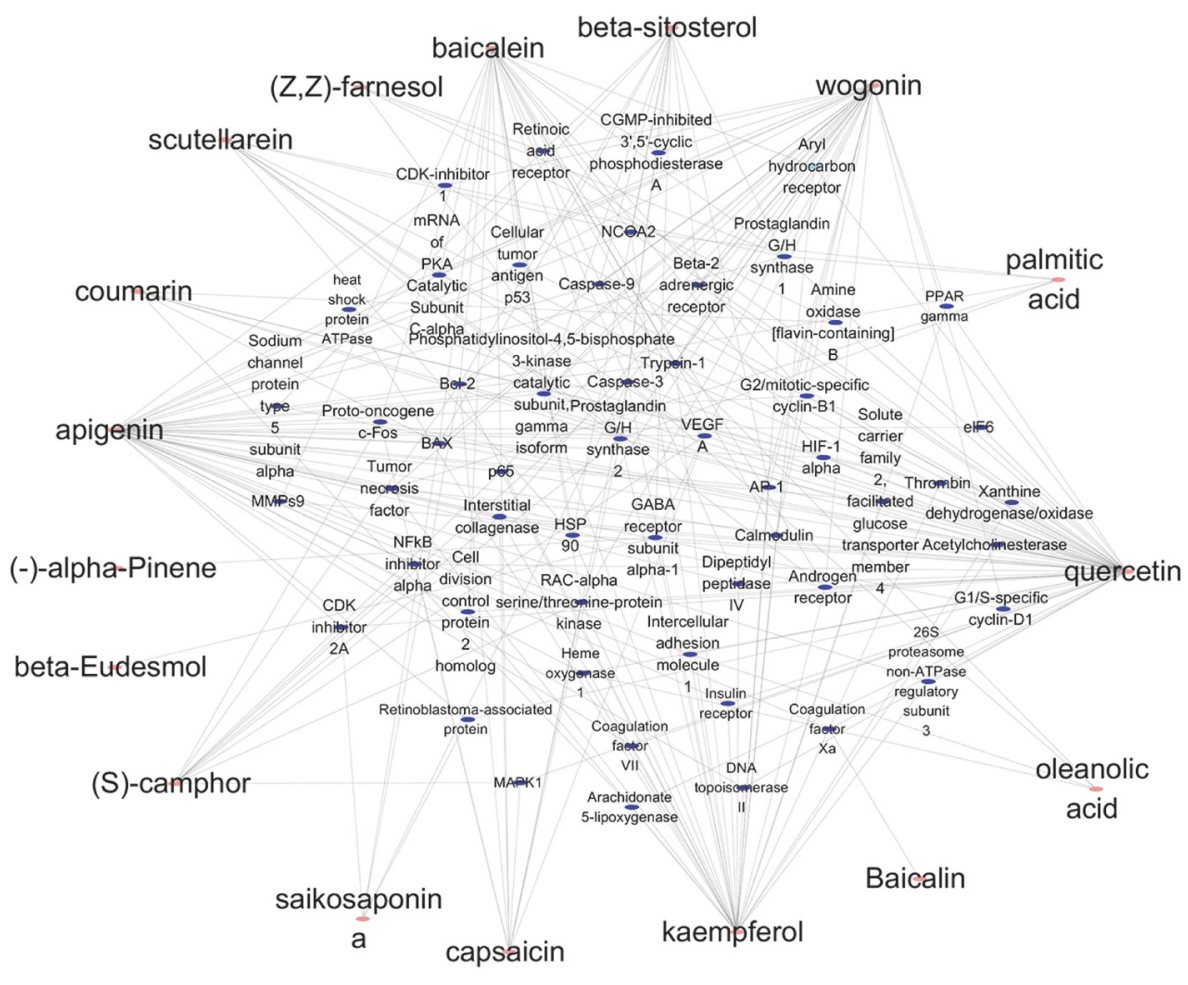

(a)

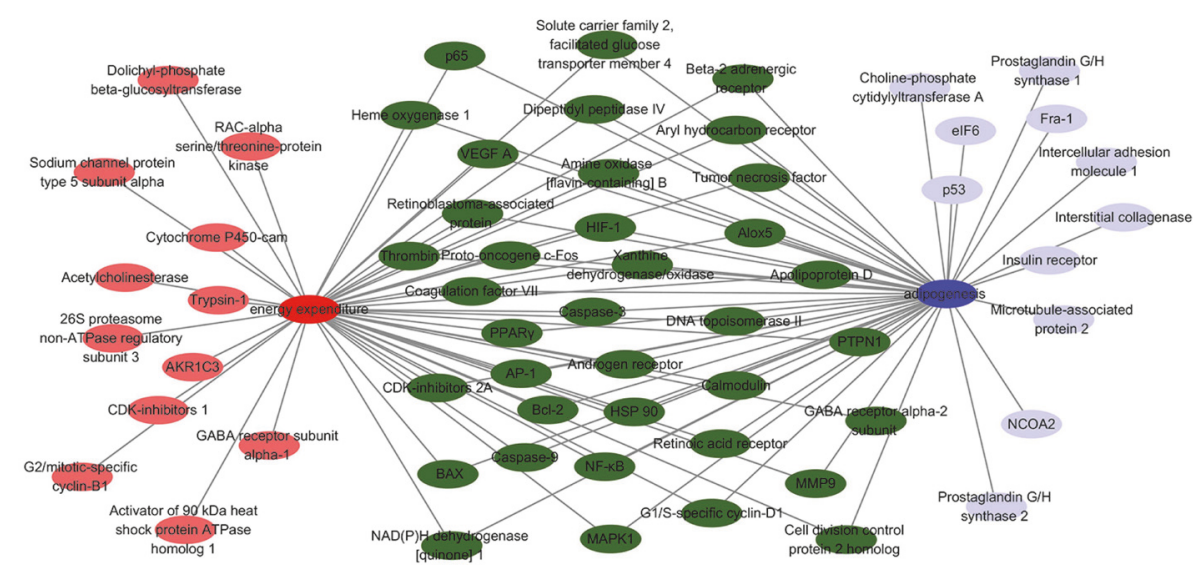

(b)

FIGURE 1: Compound-compound target network of BR and SR. (a) Compound-compound target network of BR and SR. (b) Synergistic target network of BR and SR. Blue ovals represent targets related in adipogenesis, red ovals represent targets related in energy expenditure, and green ovals represent targets related in both. 

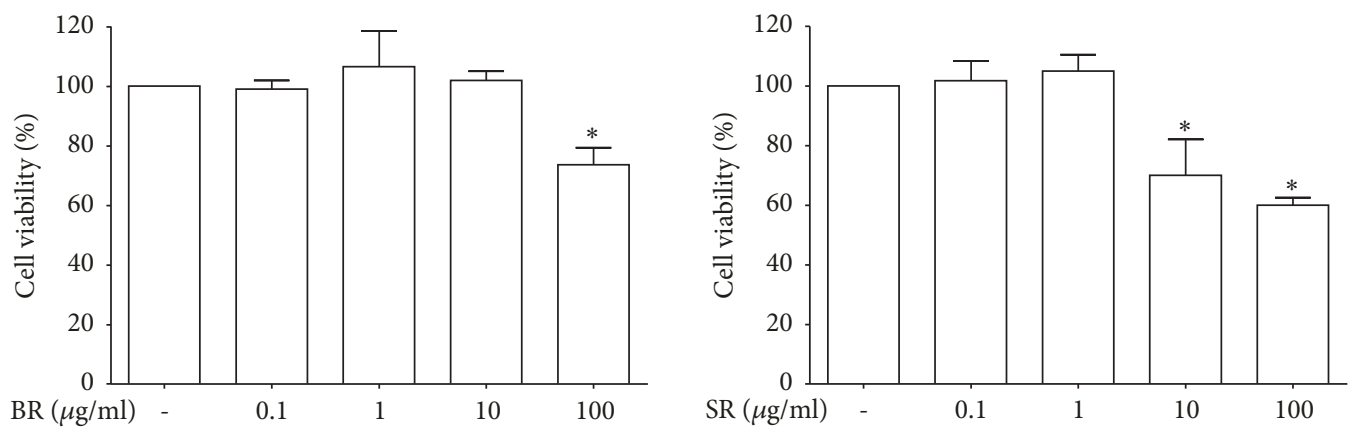

(a)
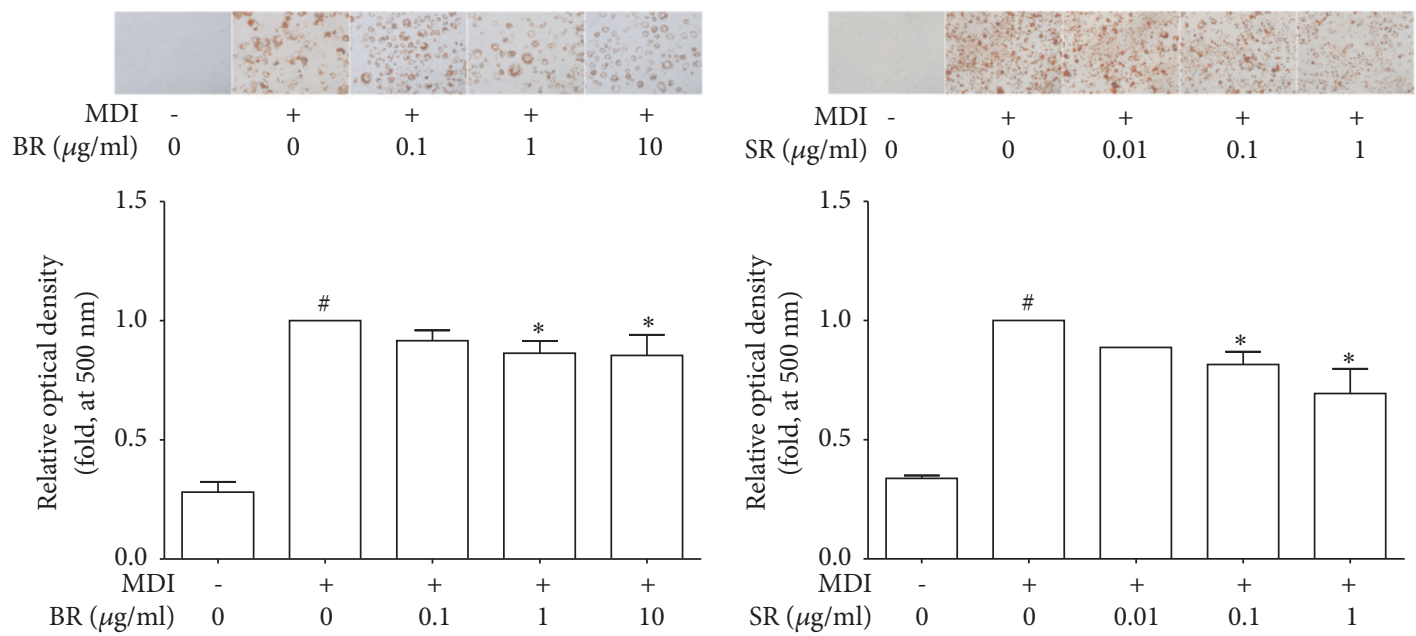

(b)
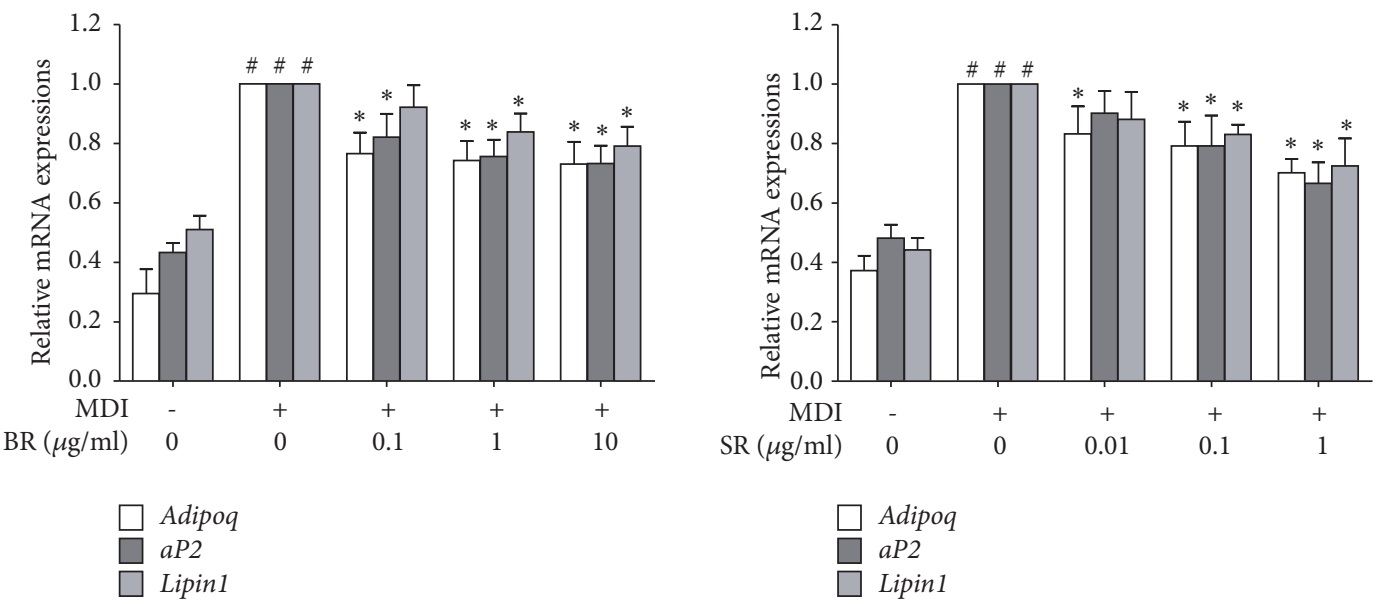

(c)

FIgURE 2: BR and SR inhibit lipid accumulation in 3T3-L1 adipocytes. (a) An MTS assay was performed in order to measure the effects of Bupleuri Radix and Scutellariae Radix on cell viability in 3T3-L1 cells. (b) An Oil Red O assay was performed in order to measure the effect of Bupleuri Radix and Scutellariae Radix on lipid accumulation in 3T3-L1 cells. (c) A Real-Time RT-PCR assay was performed in order to measure the effect of Bupleuri Radix and Scutellariae Radix on mRNA expressions of AdipoQ, aP2, and Lipin1. Gapdh mRNA was analyzed as an internal control. Experiments were repeated at least three times. Data represented are the relative expression. All values are mean \pm SEM. ${ }^{*} p<0.05$, significantly different from untreated adipocytes. BR, Bupleuri Radix; SR, Scutellariae Radix. 

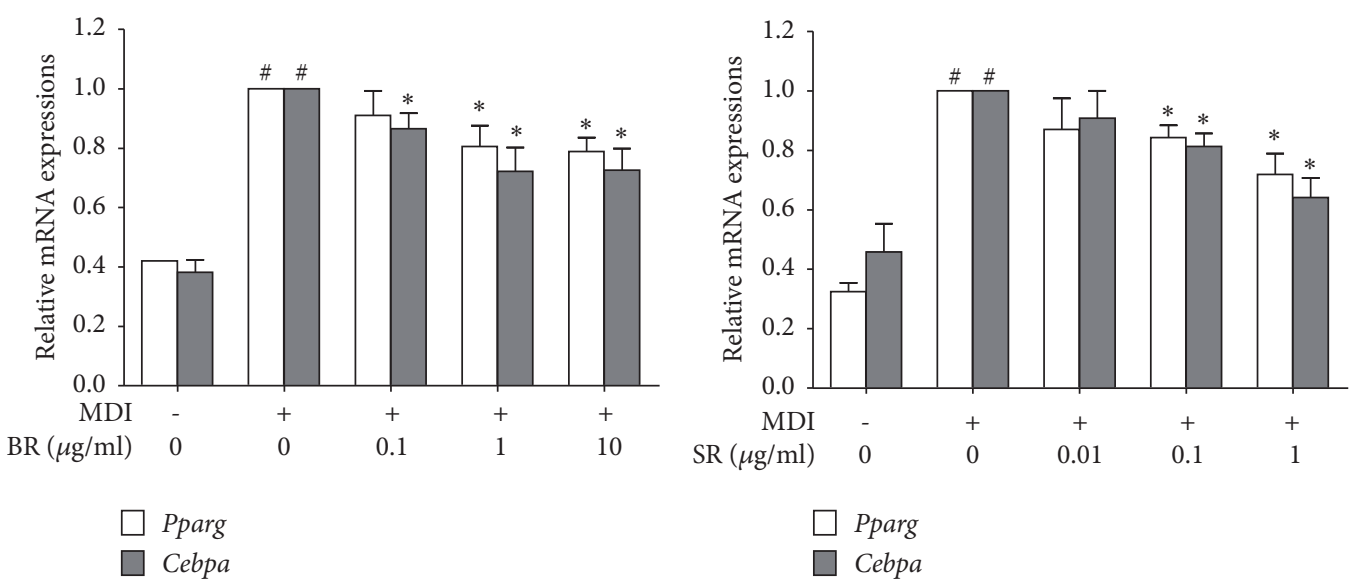

(a)
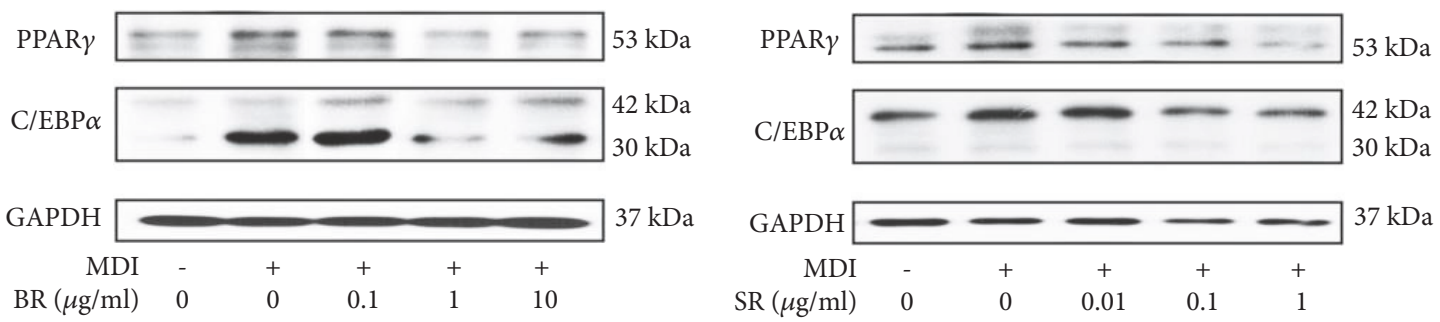

(b)
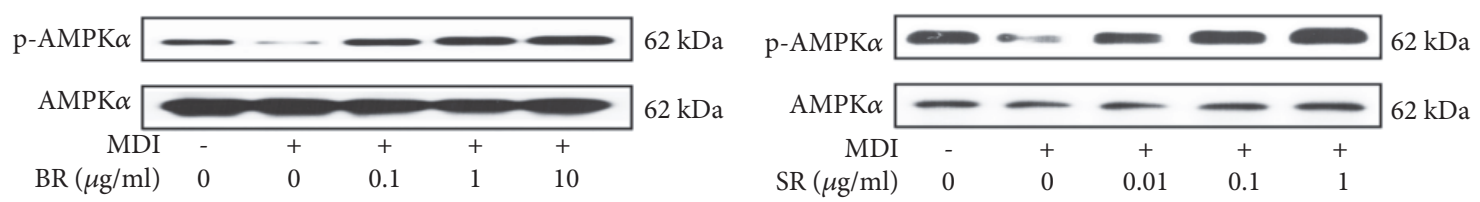

(c)

FIgURE 3: BR and SR suppress adipogenesis and increase energy expenditure in 3T3-L1 adipocytes. (a) A real-time RT-PCR assay was performed in order to measure the effect of Bupleuri Radix and Scutellariae Radix on mRNA expressions of Cebpa and Pparg. (b) A western blot assay was performed in order to measure the effect of Bupleuri Radix Scutellariae Radix on protein expressions of C/EBP $\alpha$, PPAR $\gamma$, and (c) p-AMPK $\alpha$. Gapdh mRNA was analyzed as an internal control for Real-Time RT-PCR assays. Experiments were repeated at least three times. Data represented are the relative expression. All values are mean \pm SEM. ${ }^{*} p<0.05$, significantly different from untreated adipocytes. BR, Bupleuri Radix; SR, Scutellariae Radix.

3.3. BR and SR Decrease Adipokines in 3T3-L1 Adipocytes. Real-time RT-PCR assays revealed adipokine genes including Adipoq, aP2, and Lipin1 were decreased as well (Figure 2(c)). Adiponectin, an adipokine transcripted by the Adipoq gene, promotes adipocyte differentiation by increasing $\mathrm{C} / \mathrm{EBP} \alpha$ and PPAR $\gamma$ in 3T3-Ll adipocytes [27], and also secreted adiponectins are considered as a marker to evaluate adipogenic differentiation [28]. Adipocyte protein $2(\mathrm{aP} 2)$ is a mediator of intracellular transport of fatty acids which is primarily expressed in adipocytes and macrophages [29]. Inhibition of this protein leads to a potential amelioration of obesity [30]. The role of Lipin-1, a protein with the most prominent expression in adipose tissue, skeletal muscle, and testis [31], in adipogenesis is complex. Several studies indicate its importance since the lack of Lipin-1 results in disturbed adipogenesis both in vivo and in vitro [32-34].
3.4. BR and SR Suppress Adipogenesis and Increase Energy Expenditure in 3T3-L1 Adipocytes. To investigate the action mechanism in lipid inhibition by BR and SR, we assessed the change in adipogenic factors. Two major controllers in adipogenesis, $\mathrm{C} / \mathrm{EBP} \alpha$ and PPAR $\gamma$, were significantly decreased by $\mathrm{BR}$ and SR treatment at mRNA level and protein level both (Figures 3(a) and 3(b)), suggesting suppressed adipogenesis was responsible for the antilipogenic effect of BR. Then, we evaluated the effect of BR and SR on AMP-activated protein kinase alpha (AMPK $\alpha$ ). As a result, we observed concentration-dependent increase in phosphorylation of AMPK $\alpha$ in BR- and SR-treated adipocytes (Figure 3(c)). Through these results, we could conclude that BR and SR suppress lipid accumulation in 3T3-L1 adipocytes by two action mechanisms: inhibition of adipogenesis and increase of energy expenditure. 


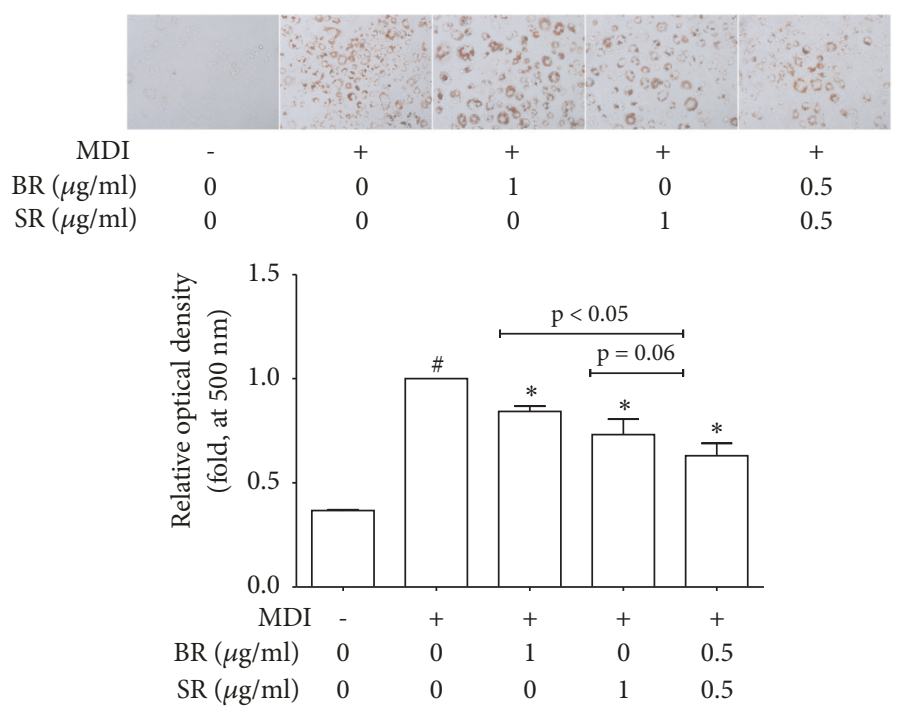

(a)

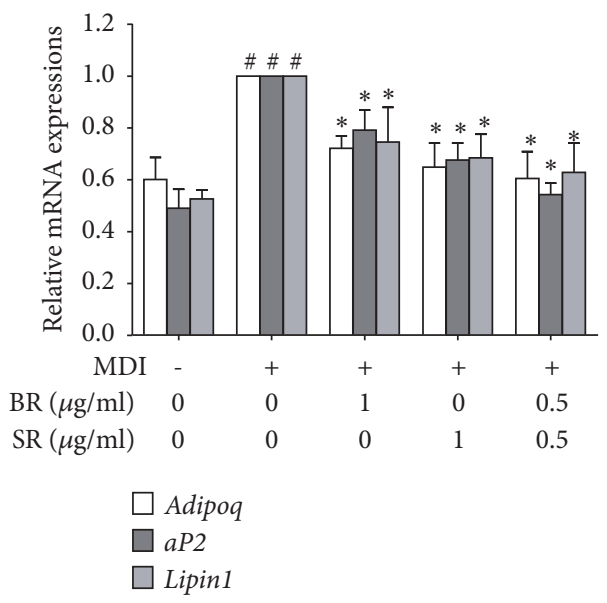

(b)

FIGURE 4: BR-SR mixture synergistically suppresses lipid accumulation in 3T3-L1 adipocytes. (a) An Oil Red O assay was performed in order to measure the effect of Bupleuri Radix, Scutellariae Radix, and their 1:1 mixture on lipid accumulation in 3T3-L1 cells. (b) A Real-Time RTPCR assay was performed in order to measure the effect of Bupleuri Radix, Scutellariae Radix, and their 1:1 mixture on mRNA expressions of AdipoQ, aP2, and Lipin1. Gapdh mRNA was analyzed as an internal control. Experiments were repeated at least three times. Data represented are the relative expression. All values are mean \pm SEM. ${ }^{*} p<0.05$, significantly different from untreated adipocytes. BR, Bupleuri Radix; SR, Scutellariae Radix.

3.5. BR-SR Mixture Synergistically Suppress Lipid Accumulation in 3T3-L1 Adipocytes. To confirm whether our hypothesis is correct or not, we then performed experiments to evaluate the synergism between $\mathrm{BR}$ and SR. BR and SR are contained in Sosiho-Tang at a 3:2 ratio (12 $\mathrm{g}$ and $8 \mathrm{~g}$, respectively), and our results on separate extracts showed $1 \mu \mathrm{g} / \mathrm{ml}$ was the most efficient concentration of SR while $1 \mu \mathrm{g} / \mathrm{ml}$ and $10 \mu \mathrm{g} / \mathrm{ml}$ of $\mathrm{BR}$ did not differ significantly. Therefore, we used BR-SR mixture at $1 \mu \mathrm{g} / \mathrm{ml}$ of which extracts were mixed at 1:1 ratio. As shown in Figure 4(a), BR-SR 1:1 mixture suppressed lipid accumulation in 3T3L1 adipocytes, whose inhibition rate was higher than BR $1 \mu \mathrm{g} / \mathrm{ml}$ or SR $1 \mu \mathrm{g} / \mathrm{ml}(p<0.05$ and $p=0.06$, respectively). Further qPCR results indicated synergistic inhibition of adipokine genes such as Adipoq, aP2, and Lipin1 (Figure $4(\mathrm{~b}))$.

3.6. BR-SR Mixture Synergistically Inhibits Adipogenesis and Increases Energy Expenditure. To investigate the effect of BRSR mixture on adipogenesis, we analyzed expression levels of Cebpa and Pparg using Real-Time RT-PCR. As in Figure 5(a), BR-SR 1:1 mixture showed higher inhibition rate in the two factors when compared to separate extracts at the same concentration. Consistently, BR-SR mixture showed synergistic inhibitory effect on protein levels of $\mathrm{C} / \mathrm{EBP} \alpha$ and $\operatorname{PPAR} \gamma$ as well (Figure 5(b)). Mixture of BR and SR also enhanced the activation of AMPK $\alpha$ (Figure 5(c)), implying that these two herbal extracts synergistically interact in two different pathways of obesity treatment: inhibiting adipogenesis and increasing energy expenditure.

\section{Discussion}

The emerging crisis of obesity impacts the world. A more serious problem is that obesity can lead to other metabolismrelated diseases, such as diabetes, cardiovascular diseases, and even cancer [35]. Current available medications for obesity are however limited; therefore the necessity of new options are growing. Nature-derived materials, in this case, have gained interest in the field of obesity treatment due to their positive effects with pharmacological safety [36]. Sosiho-Tang is a Korean medical formula composed of seven herbs: BR (12 g), SR (8 g), Pinelliae Tuber (4 g), Ginseng Radix (4 g), Zizyphi Fructus (4 g), Zingiberis Rhizoma (4 $\mathrm{g}$ ), and Glycyrrhizae Radix ( $2 \mathrm{~g}$ ). This herbal formula is a candidate for optional treatments as Yoo et al. reported its show antiobese effects in vivo and in vitro [7].

Several studies report the synergistic interaction in nature-derived materials. Our previous study has reported synergistic effect of two herbal pairs, Vertrum nigrum and Panax ginseng [37]. Zhao et al. used HFD-induced rats to show synergism between quercetin and resveratrol [38]. Another group has also reported the synergistic effect of this pair "browning" of white adipose tissue [39]. In this 


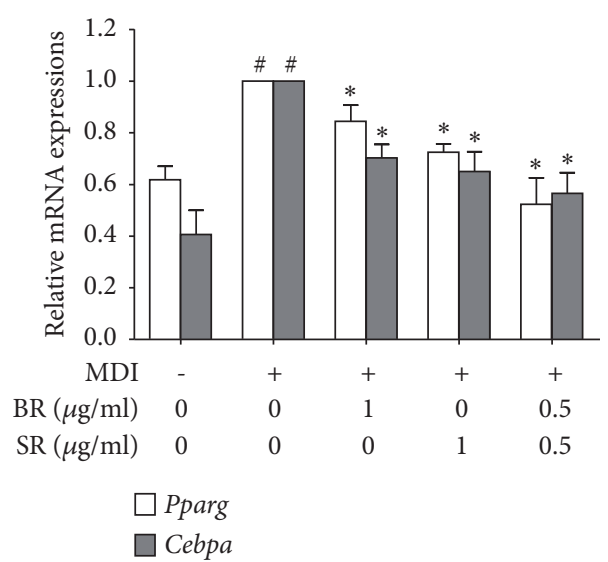

(a)

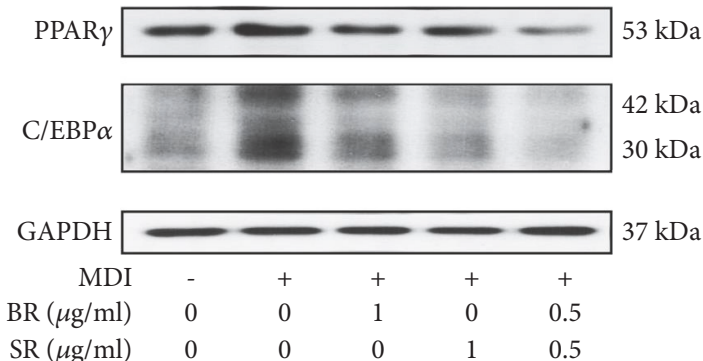

(b)

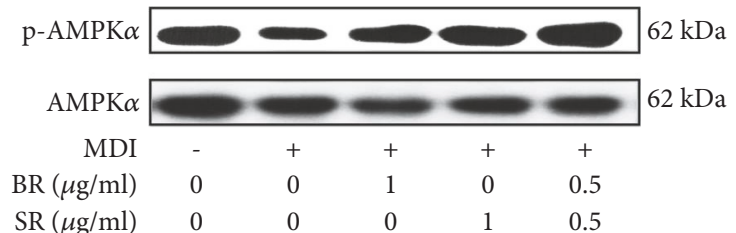

(c)

FIGURE 5: BR-SR mixture synergistically inhibits adipogenesis and increases energy expenditure. (a) A real-time RT-PCR assay was performed in order to measure the effect of Bupleuri Radix, Scutellariae Radix, and their 1:1 mixture on mRNA expressions of Cebpa and Pparg. (b) A western blot assay was performed in order to measure the effect of Bupleuri Radix, Scutellariae Radix, and their 1:1 mixture on protein expressions of $\mathrm{C} / \mathrm{EBP} \alpha, \mathrm{PPAR} \gamma$, and (c) p-AMPK $\alpha$. Gapdh mRNA was analyzed as an internal control for Real-Time RT-PCR assays. Experiments were repeated at least three times. Data represented are the relative expression. All values are mean \pm SEM. ${ }^{*} p<0.05$, significantly different from untreated adipocytes. BR, Bupleuri Radix; SR, Scutellariae Radix.

study, we first analyzed the possible synergy of BR-SR pair by a network pharmacological approach. In the compoundtarget analysis, we observed several active compounds of BR and SR regulate targets related to adipogenesis and energy expenditure.

Obesity results from accumulation of excess energy in the form of lipid in adipocytes. Adipogenesis is a process of differentiation of preadipocyte into adipocytes. Numerous transcriptional factors form a complex cascade of adipogenesis, of which process changes in shape, genes, proteins, and hormones are accompanied [40, 41]. Among them, C/EBP $\alpha$ and $\operatorname{PPAR} \gamma$ are considered to be the master regulators in adipogenesis [42]. In the current study, BR-SR mixture showed significant synergism on the inhibition of these two key adipogenic factors.

In addition to inhibiting adipogenesis, pharmacological activation of AMPK $\alpha$ is another potential pathway for obesity treatment. The energy storing WAT expresses AMPK, a serine/threonine protein kinase complex composed of three subunits (AMPK $\alpha, \beta$ and $\gamma$ ) which is activated in low energy-conditions [43]. Activated AMPK induces catalytic ATP generation while inhibiting anabolic process of energy consumption in order to maintain cellular energy homeostasis [44]. Thus, AMPK is one of the key regulators in energy homeostasis that mediate glucose and lipid metabolism to modulate energy levels [45]. Although previous studies allow the anticipation of AMPK elevation by BR [46] and SR [47, 48], the significance of our study was that, by network pharmacological analysis, a possible synergy mechanism on AMPK induction could be expected. Our results show that BR and SR extracts can activate AMPK $\alpha$, suggesting their ability to increase energy expenditure. Furthermore, the 1:1 mixture of these two herbs interacted synergistically, phosphorylating AMPK $\alpha$ at a higher level.

Besides energy expenditure, AMPK $\alpha$ is also a regulator of adipogenesis by suppressing C/EBP $\alpha$ and PPAR $\gamma$ [49]. These reports again provide substantial evidence of synergy between BR and SR on adipogenesis. However, further study is required to fully understand the synergy mechanism of this Sangsoo pair of BR and SR.

\section{Conclusion}

Taken together, we analyzed the network pharmacological action of the Sangsoo herbal pair BR and SR, the two major components of Sosiho-Tang to predict their synergistic possibility. The compound-target relevance showed that these two herbs share two major antiobese targets: adipogenesis and energy expenditure. Then we used 3T3L1 adipocytes to investigate whether the herbal pair do synergistically interact. As expected, BR-SR mixture showed synergism on suppressing intracellular lipid accumulation 
and adipokine genes, which resulted from inhibition of adipogenic factors, $\mathrm{C} / \mathrm{EBP} \alpha$ and PPAR $\gamma$, and activation of the key regulator of energy metabolism, AMPK $\alpha$. Despite the fact that further mechanism study is required and that our results lack in vivo effects of BR-SR herbal pair, our results provide experimental evidence for the Korean medical theory of Sangsoo and also suggest this herbal pair BR and SR may benefit as a potential antiobese therapeutic agent.

\section{Data Availability}

The authors will retain all data and can provide it when requested.

\section{Conflicts of Interest}

The authors declare that they have no conflicts of interest.

\section{Authors' Contributions}

The first three authors (Jueun Lee, Jinbong Park, and Hyewon Park) contributed equally to this paper and share first authorship. Jae-Young Um developed the experimental design and conducted all of the manuscript. Jueun Lee performed the network pharmacology analysis. Hyewon Park and DongHyun Youn performed the in vitro experiments. Jaehoon Lee and Seokbeom Hong provided technical and material support. Jinbong Park wrote the manuscript. All authors read and approved the final manuscript for submission.

\section{Acknowledgments}

This study was supported by the National Research Foundation of Korea (NRF-2015R1A41042399 and 2018R1D1A1B07049882) and Kyung Hee University in 2018 (KHU-1051).

\section{References}

[1] World Health Organization Media Centre, WHO Media Centre Fact Sheets: Obesity and Overweight, World Health Organization Media Centre, 2015.

[2] M. Solas, F. I. Milagro, D. Martínez-Urbistondo, M. J. Ramirez, and J. A. Martínez, "Precision obesity treatments including pharmacogenetic and nutrigenetic approaches," Trends in Pharmacological Sciences, vol. 37, no. 7, pp. 575-593, 2016.

[3] H. R. Wyatt, "Update on treatment strategies for obesity," The Journal of Clinical Endocrinology \& Metabolism, vol. 98, no. 4, pp. 1299-1306, 2013.

[4] A. L. Hopkins, "Network pharmacology," Nature Biotechnology, vol. 25, no. 10, pp. 1110-1111, 2007.

[5] A. L. Hopkins, "Network pharmacology: the next paradigm in drug discovery," Nature Chemical Biology, vol. 4, no. 11, pp. 682690, 2008.

[6] G.-b. Zhang, Q.-y. Li, Q.-1. Chen, and S.-b. Su, "Network pharmacology: a new approach for chinese herbal medicine research," Evidence-Based Complementary and Alternative Medicine, vol. 2013, Article ID 621423, 9 pages, 2013.
[7] S.-R. Yoo, M.-Y. Lee, B.-K. Kang, H.-K. Shin, and S.-J. Jeong, "Soshiho-tang aqueous extract exerts antiobesity effects in high fat diet-fed mice and inhibits adipogenesis in 3T3-L1 adipocytes," Evidence-Based Complementary and Alternative Medicine, vol. 2016, Article ID 2628901, 2016.

[8] T. F. Tzeng, H. J. Lu, S. S. Liou, C. J. Chang, and I. M. Liu, "Vinegar-baked radix bupleuri regulates lipid disorders via a pathway dependent on peroxisome-proliferator-activated receptor-alpha in high-fat-diet-induced obese rats," EvidenceBased Complementary and Alternative Medicine, vol. 2012, Article ID 827278, 12 pages, 2012.

[9] S. O. Kim, J. Y. Park, S. Y. Jeon, C. H. Yang, and M. R. Kim, "Saikosaponin a, an active compound of Radix Bupleuri, attenuates inflammation in hypertrophied 3T3-L1 adipocytes via ERK/NF- $\kappa$ B signaling pathways," International Journal of Molecular Medicine, vol. 35, no. 4, pp. 1126-1132, 2015.

[10] S.-J. Hong, J. C. Fong, and J.-H. Hwang, "Effects of crude drugs on glucose uptake in 3T3-L1 adipocytes," Kaohsiung Journal of Medical Sciences, vol. 16, no. 9, pp. 445-451, 2000.

[11] H. Lee, R. Kang, Y. Hahn et al., "Antiobesity effect of baicalin involves the modulations of proadipogenic and antiadipogenic regulators of the adipogenesis pathway," Phytotherapy Research, vol. 23, no. 11, pp. 1615-1623, 2009.

[12] D. H. Kwak, J.-H. Lee, K. H. Song, and J. Y. Ma, "Inhibitory effects of baicalin in the early stage of 3T3-L1 preadipocytes differentiation by down-regulation of PDK1/Akt phosphorylation," Molecular and Cellular Biochemistry, vol. 385, no. 1-2, pp. 257-264, 2014.

[13] L.-L. Yang, N. Xiao, J. Liu et al., "Differential regulation of baicalin and scutellarin on AMPK and Akt in promoting adipose cell glucose disposal," Biochimica et Biophysica Acta, vol. 1863, no. 2, pp. 598-606, 2017.

[14] P. Fang, M. Yu, W. Min et al., "Beneficial effect of baicalin on insulin sensitivity in adipocytes of diet-induced obese mice," Diabetes Research and Clinical Practice, vol. 139, pp. 262-271, 2018.

[15] M.-H. Cha, I.-C. Kim, B.-H. Lee, and Y. Yoon, "Baicalein inhibits adipocyte differentiation by enhancing COX-2 expression," Journal of Medicinal Food, vol. 9, no. 2, pp. 145-153, 2006.

[16] Y. Nakao, H. Yoshihara, K. Fujimori, and H. Xu, "Suppression of very early stage of adipogenesis by baicalein, a plant-derived flavonoid through reduced Akt-C/EBP $\alpha$-GLUT4 signalingmediated glucose uptake in 3T3-L1 adipocytes," PLoS ONE, vol. 11, no. 9, Article ID e0163640, 2016.

[17] E.-J. Bak, J. Kim, Y. H. Choi et al., "Wogonin ameliorates hyperglycemia and dyslipidemia via PPAR $\alpha$ activation in $\mathrm{db} / \mathrm{db}$ mice," Clinical Nutrition, vol. 33, no. 1, pp. 156-163, 2014.

[18] T. Yang, H. Liu, B. Zhao et al., "Wogonin enhances intracellular adiponectin levels and suppresses adiponectin secretion in 3T3L1 adipocytes," Endocrine Journal, vol. 64, no. 1, pp. 15-26, 2017.

[19] J. Chen, J. Liu, and Y. Wang, "Wogonin mitigates nonalcoholic fatty liver disease via enhancing PPAR $\alpha /$ AdipoR2, in vivo and in vitro," Biomedicine \& Pharmacotherapy, vol. 91, pp. 621-631, 2017.

[20] R. Jin, B. Zhang, C.-M. Xue, S.-M. Liu, Q. Zhao, and K. Li, "Classification of 365 Chinese medicines in Shennong's Materia Medica Classic based on a semi-supervised incremental clustering method," Journal of Chinese Integrative Medicine, vol. 9, no. 6, pp. 665-674, 2011.

[21] D. Merico, D. Gfeller, and G. D. Bader, "How to visually interpret biological data using networks," Nature Biotechnology, vol. 27, no. 10, pp. 921-924, 2009. 
[22] J. Park, Y.-D. Jeon, H.-L. Kim et al., "Veratri Nigri Rhizoma et Radix (Veratrum nigrum L.) and its constituent jervine prevent adipogenesis via activation of the LKB1-AMPKalphaACC axis in vivo and in vitro," Evidence-Based Complementary and Alternative Medicine, vol. 2016, Article ID 8674397, 12 pages, 2016.

[23] Y. Jung, J. Park, H. Kim et al., "Vanillic acid attenuates obesity ," The FASEB Journal, vol. 32, no. 3, pp. 1388-1402, 2018.

[24] M.-Y. Jeong, H.-L. Kim, J. Park et al., "Rubi Fructus (Rubus coreanus) activates the expression of thermogenic genes in vivo and in vitro," International Journal of Obesity, vol. 39, no. 3, pp. 464-456, 2015.

[25] M.-Y. Jeong, J. Park, D.-H. Youn et al., "Albiflorin ameliorates obesity by inducing thermogenic genes via AMPK and PI3K/AKT in vivo and in vitro," Metabolism - Clinical and Experimental, vol. 73, pp. 85-99, 2017.

[26] H. Kim, Y. Jung, J. Park et al., "Farnesol has an anti-obesity effect in high-fat diet-induced obese mice and induces the development of beige adipocytes in human adipose tissue derived-mesenchymal stem cells," Frontiers in Pharmacology, vol. 8, article 654, 2017.

[27] Y. Fu, N. Luo, R. L. Klein, and W. Timothy Garvey, "Adiponectin promotes adipocyte differentiation, insulin sensitivity, and lipid accumulation," Journal of Lipid Research, vol. 46, no. 7, pp. 13691379, 2005.

[28] E. Martella, C. Bellotti, B. Dozza, S. Perrone, D. Donati, and E. Lucarelli, "Secreted adiponectin as a marker to evaluate in vitro the adipogenic differentiation of human mesenchymal stromal cells," Cytotherapy, vol. 16, no. 11, pp. 1476-1485, 2014.

[29] L. Sun, A. C. Nicholson, D. P. Hajjar, A. M. Gotto Jr., and J. Han, "Adipogenic differentiating agents regulate expression of fatty acid binding protein and CD36 in the J744 macrophage cell line," Journal of Lipid Research, vol. 44, no. 10, pp. 1877-1886, 2003.

[30] K. Maeda, H. Cao, K. Kono et al., "Adipocyte/macrophage fatty acid binding proteins control integrated metabolic responses in obesity and diabetes," Cell Metabolism, vol. 1, no. 2, pp. 107-119, 2005.

[31] M. Péterfy, J. Phan, P. Xu, and K. Reue, "Lipodystrophy in the fld mouse results from mutation of a new gene encoding a nuclear protein, lipin," Nature Genetics, vol. 27, no. 1, pp. 121-124, 2001.

[32] K. Reue, P. Xu, X.-P. Wang, and B. G. Slavin, "Adipose tissue deficiency, glucose intolerance, and increased atherosclerosis result from mutation in the mouse fatty liver dystrophy (fld) gene," Journal of Lipid Research, vol. 41, no. 7, pp. 1067-1076, 2000.

[33] P. Zhang, K. Takeuchi, L. S. Csaki, and K. Reue, "Lipin1 phosphatidic phosphatase activity modulates phosphatidate levels to promote peroxisome proliferator-activated receptor $\gamma$ $(\mathrm{PPAR} \gamma)$ gene expression during adipogenesis," The Journal of Biological Chemistry, vol. 287, no. 5, pp. 3485-3494, 2012.

[34] H. Sembongi, M. Miranda, G.-S. Han et al., "Distinct roles of the phosphatidate phosphatases lipin 1 and 2 during adipogenesis and lipid droplet biogenesis in 3T3-L1 cells," The Journal of Biological Chemistry, vol. 288, no. 48, pp. 34502-34513, 2013.

[35] NCD Risk Factor Collaboration, "Worldwide trends in bodymass index, underweight, overweight, and obesity from 1975 to 2016: a pooled analysis of 2416 population-based measurement studies in 128.9 million children, adolescents, and adults," Lancet, vol. 390, no. 10113, pp. 2627-2642, 2016.

[36] Y. Liu, M. Sun, H. Yao, Y. Liu, and R. Gao, "Herbal medicine for the treatment of obesity: an overview of scientific evidence from
2007 to 2017," Evidence-Based Complementary and Alternative Medicine, vol. 2017, Article ID 8943059, 17 pages, 2017.

[37] J. Park, Y.-D. Jeon, H.-L. Kim et al., "Interaction of Veratrum nigrum with Panax ginseng against obesity: A Sang-ban relationship," Evidence-Based Complementary and Alternative Medicine, vol. 2013, Article ID 732126, 13 pages, 2013.

[38] L. Zhao, F. Cen, F. Tian et al., "Combination treatment with quercetin and resveratrol attenuates high fat diet-induced obesity and associated inflammation in rats via the AMPK $\alpha 1 /$ SIRT1 signaling pathway," Experimental and Therapeutic Medicine, vol. 146, pp. 5942-5948, 2017.

[39] N. Arias, C. Picó, M. Teresa Macarulla et al., "A combination of resveratrol and quercetin induces browning in white adipose tissue of rats fed an obesogenic diet," Obesity, vol. 25, no. 1, pp. 111-121, 2017.

[40] H. Koutnikova and J. Auwerx, "Regulation of adipocyte differentiation," Annals of Medicine, vol. 33, no. 8, pp. 556-561, 2001.

[41] E. D. Rosen and O. A. MacDougald, "Adipocyte differentiation from the inside out," Nature Reviews Molecular Cell Biology, vol. 7, no. 12, pp. 885-896, 2006.

[42] R. F. Morrison and S. R. Farmer, "Hormonal signaling and transcriptional control of adipocyte differentiation," Journal of Nutrition, vol. 130, no. 12, pp. 3116S-3121S, 2000.

[43] M. C. Towler and D. G. Hardie, "AMP-activated protein kinase in metabolic control and insulin signaling," Circulation Research, vol. 100, no. 3, pp. 328-341, 2007.

[44] D. G. Hardie, F. A. Ross, and S. A. Hawley, "AMPK: a nutrient and energy sensor that maintains energy homeostasis," Nature Reviews Molecular Cell Biology, vol. 13, no. 4, pp. 251-262, 2012.

[45] R. B. Ceddia, "The role of AMP-activated protein kinase in regulating white adipose tissue metabolism," Molecular and Cellular Endocrinology, vol. 366, no. 2, pp. 194-203, 2013.

[46] Z. Zhen, B. Chang, M. Li et al., "Anti-diabetic effects of a coptis chinensis containing new traditional Chinese medicine formula in type 2 diabetic rats," American Journal of Chinese Medicine, vol. 39, no. 1, pp. 53-63, 2011.

[47] T. Wang, H. Jiang, S. Cao et al., "Baicalin and its metabolites suppresses gluconeogenesis through activation of AMPK or AKT in insulin resistant HepG-2 cells," European Journal of Medicinal Chemistry, vol. 141, pp. 92-100, 2017.

[48] Q. Chen, M. Liu, H. Yu et al., "Scutellaria baicalensis regulates FFA metabolism to ameliorate NAFLD through the AMPK-mediated SREBP signaling pathway," Journal of Natural Medicines, vol. 72, no. 3, pp. 655-666, 2018.

[49] Y. Dagon, Y. Avraham, and E. M. Berry, "AMPK activation regulates apoptosis, adipogenesis, and lipolysis by eIF $2 \alpha$ in adipocytes," Biochemical and Biophysical Research Communications, vol. 340, no. 1, pp. 43-47, 2006. 


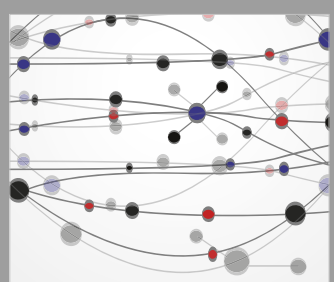

The Scientific World Journal
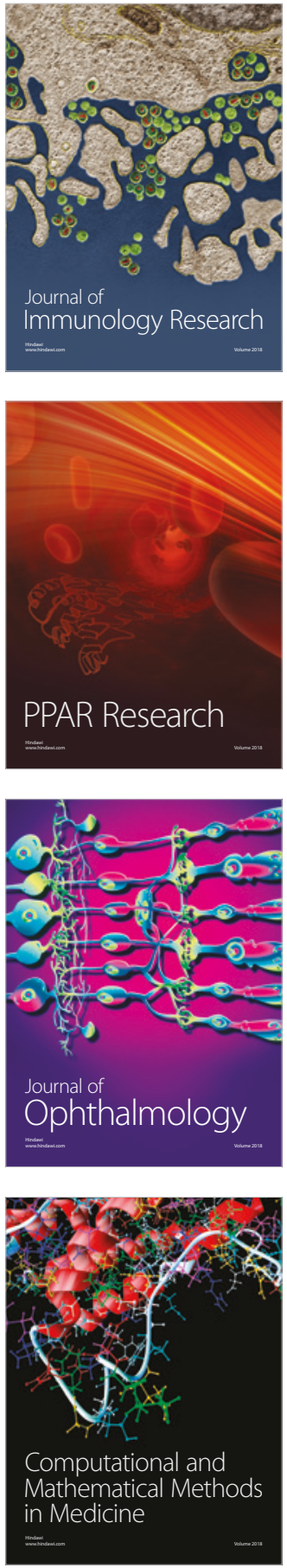

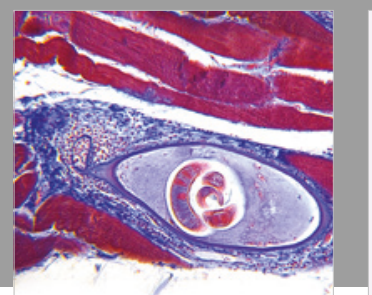

Gastroenterology Research and Practice

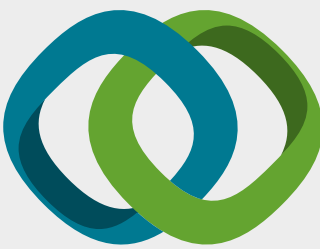

\section{Hindawi}

Submit your manuscripts at

www.hindawi.com
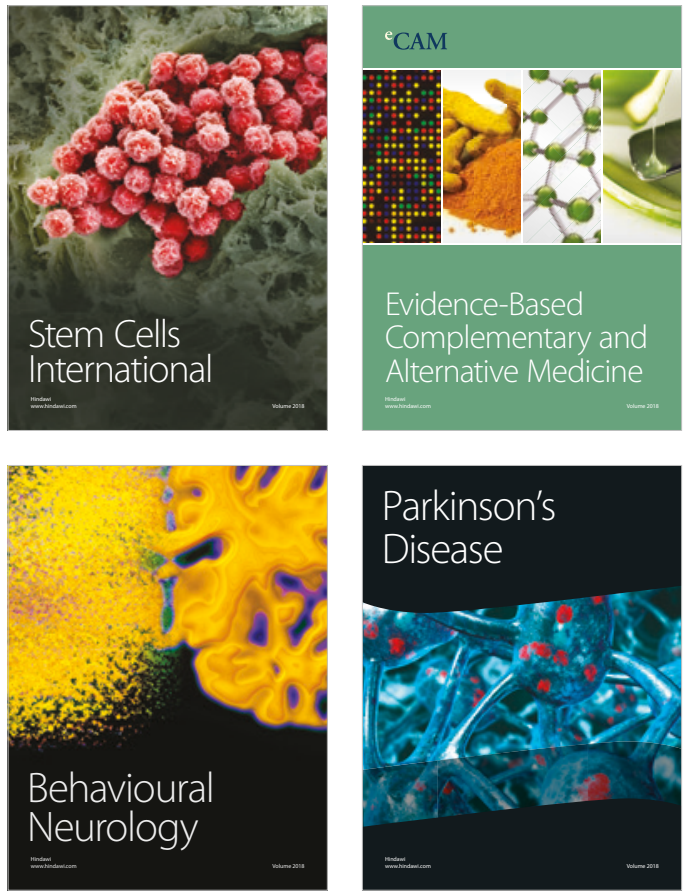

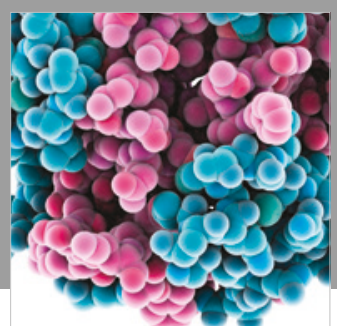

ournal of

Diabetes Research

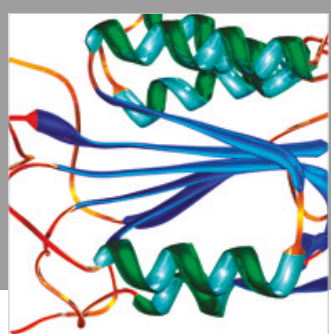

Disease Markers
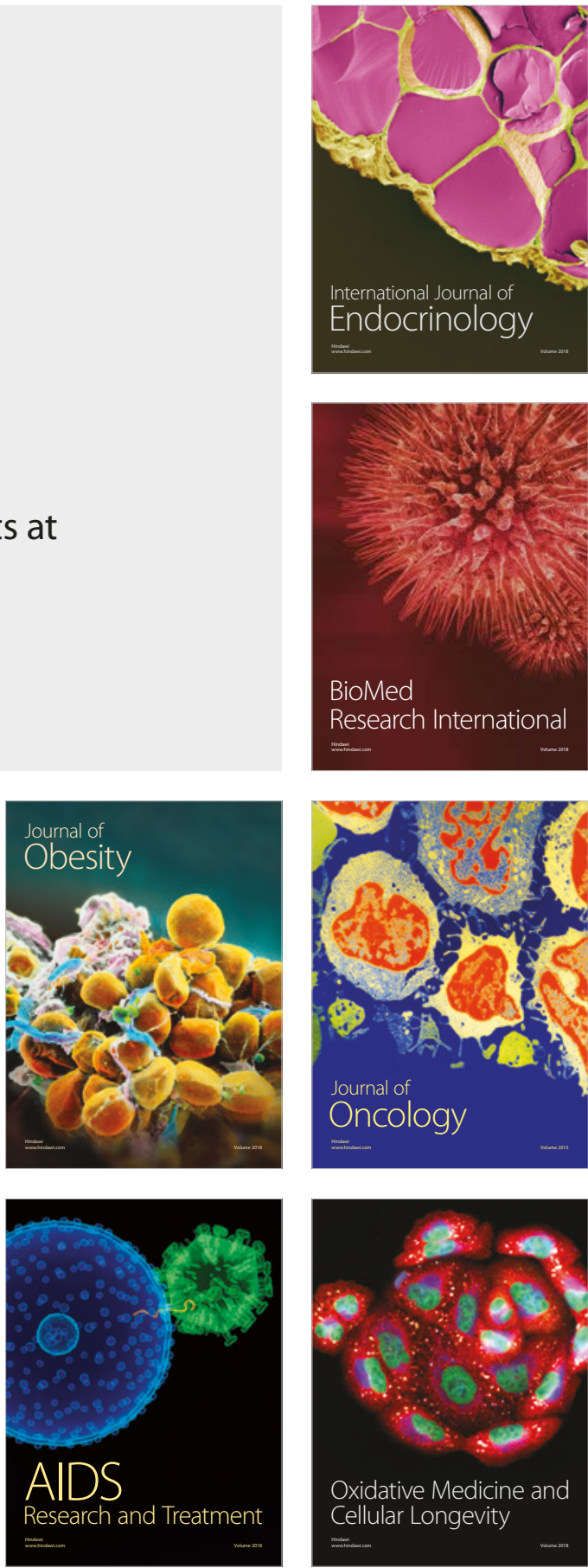